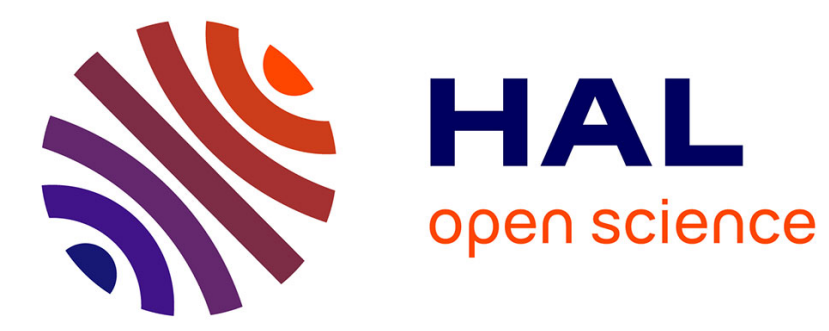

\title{
Post processing for the vector finite element method: from edge to nodal values
}

\author{
Christian Vollaire, François Musy, Ronan Perrussel
}

\section{To cite this version:}

Christian Vollaire, François Musy, Ronan Perrussel. Post processing for the vector finite element method: from edge to nodal values. COMPEL: The International Journal for Computation and Mathematics in Electrical and Electronic Engineering, 2005, 24, Number 4, p.: 1274-1283. 10.1108/03321640510615607 . hal-00113507

\section{HAL Id: hal-00113507 https://hal.science/hal-00113507}

Submitted on 13 Nov 2006

HAL is a multi-disciplinary open access archive for the deposit and dissemination of scientific research documents, whether they are published or not. The documents may come from teaching and research institutions in France or abroad, or from public or private research centers.
L'archive ouverte pluridisciplinaire HAL, est destinée au dépôt et à la diffusion de documents scientifiques de niveau recherche, publiés ou non, émanant des établissements d'enseignement et de recherche français ou étrangers, des laboratoires publics ou privés. 


\section{Post Processing for the Vector Finite Element Method: from Edge to Nodal Values}

Christian VOLLAIRE : CEGELY, UMR CNRS 5005, christian.vollaire@ec-lyon.fr, Ecole Centrale de Lyon, 69134 Ecully, France.

François MUSY : MAPLY, UMR CNRS 5585, francois.musy@ec-lyon.fr, Ecole Centrale de Lyon, 69134 Ecully, France.

Ronan PERRUSSEL: MAPLY, UMR CNRS 5585, CEGELY, UMR CNRS 5005 ronan.perrussel@ec-lyon.fr, Ecole Centrale de Lyon, 69134 Ecully, France.

\section{Purpose}

Propose post processing methods for the edge finite element method on a tetrahedral mesh. They make it possible to deduce vector values on the vertices from scalar values defined on the edges of the tetrahedra.

\section{Approach}

The new proposed techniques are based on a least squares formulation leading to a sparse matrix system to be solved. They are compared in terms of accuracy and CPU time on a FE formulation for open boundary - frequency domain problems.

\section{Findings}


A significant improvement of vector values accuracy on the vertices of the tetrahedra is obtained compared to a classical approach with a very small additional computation time.

\section{Originality}

This work presents techniques allowing:

- To obtain the values at the initial nodes of the mesh and not inside the tetrahedra

- To take into account the discontinuity to the interface between two media of different electromagnetic properties.

\section{Keywords}

Edge elements, finite element method, post processing

\section{Research paper}




\section{Introduction}

Edge-based finite elements (FE) are useful in modeling electromagnetic phenomena because of their correct physical sense. Furthermore, it has been shown that better approximation of the solution may be obtained compared to nodal-based FE [Webb, 1993]. The incomplete first order edge finite element is commonly used in electromagnetic modeling. The degrees of freedom are line integrals along the edges in the mesh. However, knowledge of the nodal field values remains necessary for various reasons. Maximal values located at the interfaces can be required to predict possible electric breakdowns. Nodal values may be necessary to achieve some additional computation: induced currents in the conductors, dual field, source term for coupled problem (magneto thermal), ... [Zhao et al, 2000, Sekkak et al, 1994]. Post processors for visualization are usually based on the nodal representation of the fields.

For vector finite elements, two techniques are commonly used to compute nodal values from degrees of freedom [Volakis et al, 2000]:

- Only nodal values inside an element are computed from its edge values. However, this method doesn't give a unique value on its boundary, namely on the vertices.

- An average value of the nodal field is evaluated on each node and for each region by taking into account the contribution of all the elements connected to the considered node [Dibben et al, 1997].

The objective of this paper is to propose a method to compute accurate nodal values at the vertices of a tetrahedral mesh. Our approach is validated on a FE formulation for open boundary frequency domain problems. Spatial discretization is achieved using incomplete first order tetrahedral edge elements [Yao Bi, 1996].

\section{Reference method}


A first and simplest method consists in computing local node values on each element of the mesh from the edge values by means of the local edge basis. As explained by (1), a value at a node is then obtained as an arithmetic mean involving the elements containing the node. For a node at the interface between two regions, two values are obtained by averaging the field values separately in each region. It is taken as reference in the following (method 1).

$$
\mathrm{E}(\mathrm{m})=\frac{\sum_{\mathrm{T} \ni \mathrm{m}} \mathrm{E}_{\mathrm{T}}(\mathrm{m})}{\mathrm{n}_{\mathrm{T}}} \text { with } \mathrm{E}_{\mathrm{T}}(\mathrm{m})=\sum_{\mathrm{i}=1}^{6} \alpha_{\mathrm{i}} \mathrm{p}_{\mathrm{i}}(\mathrm{m})
$$

$\mathrm{m}$ is a node which belongs to $\mathrm{n}_{T}$ elements. Associated to edge $\mathrm{e}_{\mathrm{i}}$ of tetrahedron $\mathrm{T}, \mathrm{p}_{\mathrm{i}}$ is the local shape function with $\alpha_{i}$ as degree of freedom.

\section{New approach}

Let a complex vector field $\mathrm{E}$ be obtained from some computations with $\mathrm{H}(\mathrm{curl} ; \Omega)$ conforming finite elements on a tetrahedral mesh $\tau_{\mathrm{h}}$ of a bounded domain $\Omega$ of $\mathbb{R}^{3}$. We are seeking a "good representation" of $\mathrm{E}$ by a continuous vector field $\mathrm{E}^{*}$ on $\tau_{\mathrm{h}}$. This problem may be formalised by introducing the minimization problem:

Find a vector field $\mathrm{E}^{*} \in \mathrm{V}_{\mathrm{h}}$ which minimizes $\|\mathrm{E}-\mathrm{E}\|$ for all $\mathrm{E}$ in $\mathrm{V}_{\mathrm{h}}$ 
$\mathrm{V}_{\mathrm{h}}$ is the nodal conforming FE space of degree 1 defined on $\tau_{\mathrm{h}}$ and \|\| is a norm induced by a scalar product in $\mathrm{H}(\operatorname{curl} ; \Omega)$. Vector field $\mathrm{E}$ being given from incomplete first order edge elements, it can be decomposed in the edge basis denoted $\left(\mathrm{w}_{\mathrm{e}}\right)_{\mathrm{e} \in \varepsilon_{\mathrm{h}}}$, as:

$$
\mathrm{E}=\sum_{\mathrm{e} \in \varepsilon_{\mathrm{h}}} \alpha_{\mathrm{ew}} \text { with } \alpha_{\mathrm{e}}=\int_{\mathrm{e}} \text { E.t }: \text { circulation of } \mathrm{E} \text { along edge } \mathrm{e}
$$

$\varepsilon_{\mathrm{h}}$ denotes the set of the edges in $\tau_{\mathrm{h}}$ and $\mathrm{t}$ is an oriented tangent unit vector on edge e. So it seems appropriate to introduce a norm deduced by the following scalar product:

$$
\left\langle\mathrm{E}, \mathrm{E}^{\prime}\right\rangle_{\theta}=(1-\theta) \int_{\Omega} \mathrm{E} \cdot \overline{\mathrm{E}^{\prime}}+\theta \sum_{\mathrm{e} \in \varepsilon_{\mathrm{h}}}\left(\int_{\mathrm{e}} \mathrm{E} \cdot \mathrm{t}\right)\left(\int_{\mathrm{e}} \overline{\mathrm{E}}^{\prime} . \mathrm{t}\right)
$$

$\theta$ is a weight such that $0 \leq \theta<1$. The limit case $\theta=1$, for which the bilinear form $\langle,\rangle_{\theta}$ does not remain a scalar product on $\mathrm{H}(\operatorname{curl} ; \Omega)$, is tackled in the later.

By definition of the norm deduced by (4), the gap between vector fields $E$ and $E$ is

$$
\|\mathrm{E}-\mathrm{E}\|_{\theta}=\left[(1-\theta) \int_{\Omega}|\mathrm{E}-\mathrm{E}|^{2}+\theta \sum_{\mathrm{e} \in \varepsilon_{\mathrm{h}}}\left|\int_{\mathrm{e}}(\mathrm{E}-\mathrm{E}) \cdot \mathrm{t}\right|^{2}\right]^{1 / 2}
$$

By minimizing $\|\mathrm{E}-\mathrm{E}\|_{\theta}$, according to the choice of the weight $\theta$, good approximation $\mathrm{E}^{*}$ of E as well as preserving of the "circulation" along the edges may be expected. 


\section{The linear system to solve}

For the norm defined by (5) with $0 \leq \theta<1$, the minimization problem (2) is a least squares problem with a unique solution $\mathrm{E}^{*}$. Moreover vector field $\mathrm{E}^{*}$ is the orthogonal projection of $\mathrm{E}$ into the space $\mathrm{V}_{\mathrm{h}}$ for the scalar product (4). The unknown field $\mathrm{E}^{*}$ can be decomposed as:

$$
\mathrm{E}^{*}=\sum_{\mathrm{i}=1}^{\mathrm{N}_{\mathrm{h}}} \xi_{\mathrm{i}} \mathrm{v}_{\mathrm{i}}
$$

where $\left(v_{i}\right), i=1$ to $N_{h}$ (the nodal space dimension) is the nodal vector basis of the FE space $V_{h}$ and the unknown vector $\xi=\left(\xi_{\mathrm{i}}\right), \mathrm{i}=1$ to $\mathrm{N}_{\mathrm{h}}$ contains the values of each component of the field $\mathrm{E}^{*}$ on the vertices of the mesh $\tau_{\mathrm{h}}$. Writing that $\mathrm{E}^{*}-\mathrm{E}$ is orthogonal to any test function $\mathrm{v}_{\mathrm{i}}$ gives $\xi$ as solution to a linear system: $A_{\theta} \xi=b_{\theta}$. The matrix $A_{\theta}$, the Gram matrix associated to the scalar product $\langle,\rangle_{\theta}$, is given by:

$$
\left(A_{\theta}\right)_{i j}=\left\langle v_{i}, v_{j}\right\rangle_{\theta} i, j=1 \text { to } N_{h}
$$

Then, $A_{\theta}$ is real symmetric and positive definite for $0 \leq \theta<1$. The right-hand side $b_{\theta}$ is given by:

$$
\left(b_{\theta}\right)_{i}=\left\langle E, v_{i}\right\rangle_{\theta}, i=1 \text { to } N_{h}
$$

Numbering the unknowns according to the 3 components on the axes, the nodal basis can be decomposed as 


$$
\left[\begin{array}{c}
\varphi_{i} \\
0 \\
0
\end{array}\right] \mathrm{i}=1 \text { to } n,\left[\begin{array}{c}
0 \\
\varphi_{i} \\
0
\end{array}\right] \mathrm{i}=1 \text { to } n,\left[\begin{array}{c}
0 \\
0 \\
\varphi_{i}
\end{array}\right] \mathrm{i}=1 \text { to } n
$$

where $\varphi_{i}, i=1$ to $n$ is the scalar nodal basis and $n$ the number of vertices. It induces a partition of the matrix $A_{\theta}$ into a 3 by 3 block matrix with $9(n \times n)$ blocks:

$$
A_{\theta}=\left[\begin{array}{ccc}
A_{\theta}^{X X} & A_{\theta}^{X Y} & A_{\theta}^{X Z} \\
A_{\theta}^{Y X} & A_{\theta}^{Y Y} & A_{\theta}^{Y Z} \\
A_{\theta}^{Z X} & A_{\theta}^{Z Y} & A_{\theta}^{Z Z}
\end{array}\right]
$$

and similarly for $\mathrm{b}_{\theta}$ and $\xi$ with $3(\mathrm{n} \times 1)$ blocks:

$$
\mathrm{b}_{\theta}=\left[\begin{array}{c}
\mathrm{b}_{\theta}^{\mathrm{X}} \\
\mathrm{b}_{\theta}^{\mathrm{Y}} \\
\mathrm{b}_{\theta}^{\mathrm{Z}}
\end{array}\right] \xi=\left[\begin{array}{l}
\xi^{\mathrm{X}} \\
\xi^{\mathrm{Y}} \\
\xi^{\mathrm{Z}}
\end{array}\right]
$$

\section{Entries of the matrix}

It is convenient to split the system as

$$
A_{\theta}=(1-\theta) A_{0}+\theta A_{1} \text { and } b_{\theta}=(1-\theta) b_{0}+\theta b_{1}
$$

Then from (4) and (7), it comes 


$$
\begin{aligned}
& \left(\mathrm{A}_{0}\right)_{\mathrm{ij}}=\int_{\Omega} \mathrm{v}_{\mathrm{i}} \cdot \mathrm{v}_{\mathrm{j}}, \mathrm{i}, \mathrm{j}=1 \text { to } \mathrm{N}_{\mathrm{h}} \\
& \left(\mathrm{A}_{1}\right)_{\mathrm{ij}}=\sum_{\mathrm{e} \in \varepsilon_{\mathrm{h}}}\left(\int_{\mathrm{e}} \mathrm{v}_{\mathrm{i}} \cdot \mathrm{t}\right)\left(\int_{\mathrm{e}} \mathrm{v}_{\mathrm{j}} \cdot \mathrm{t}\right), \mathrm{i}, \mathrm{j}=1 \text { to } \mathrm{N}_{\mathrm{h}}
\end{aligned}
$$

and from (4) and (8)

$$
\begin{aligned}
& \left(\mathrm{b}_{0}\right)_{\mathrm{i}}=\int_{\Omega} \mathrm{E} \cdot \mathrm{v}_{\mathrm{i}}, \mathrm{i}=1 \text { to } \mathrm{N}_{\mathrm{h}} \\
& \left(\mathrm{b}_{1}\right)_{\mathrm{i}}=\sum_{\mathrm{e} \in \varepsilon_{\mathrm{h}}}\left(\int_{\mathrm{e}}^{\mathrm{E}} \mathrm{t}\right)\left(\int_{\mathrm{e}} \mathrm{v}_{\mathrm{i}} \cdot \mathrm{t}\right), \mathrm{i}=1 \text { to } \mathrm{N}_{\mathrm{h}}
\end{aligned}
$$

From (9) and (13), it is easy to check that $\mathrm{A}_{0}$ is given by:

$$
\mathrm{A}_{0}=\left(\begin{array}{ccc}
\mathrm{M} & 0 & 0 \\
0 & \mathrm{M} & 0 \\
0 & 0 & \mathrm{M}
\end{array}\right)
$$

where $M$ is the usual $(n \times n)$ mass matrix defined by:

$$
\mathrm{M}_{\mathrm{ij}}=\int_{\Omega} \varphi_{\mathrm{i}} \varphi_{\mathrm{j}}, \mathrm{i}, \mathrm{j}=1 \text { to } \mathrm{n}
$$

$A_{1}$ is not a block diagonal matrix but it is easy to see from (19) that each block is as sparse as M. Associated to each vertex $a_{i}$ of the mesh, we introduce the sets of edges 
$\varepsilon_{\mathrm{h}}^{\mathrm{i}}=\left\{\mathrm{e} \in \varepsilon_{\mathrm{h}} /\right.$ vertex $\mathrm{a}_{\mathrm{i}}$ is an extremity of edge $\left.\mathrm{e}\right\}$. Then denoting by $\mathrm{e}_{\mathrm{x}}, \mathrm{e}_{\mathrm{y}}, \mathrm{e}_{\mathrm{z}}$ the 3 components on the axes of an oriented edge e, from

$$
\int_{\mathrm{e}} \varphi_{\mathrm{i}} \mathrm{t}_{\mathrm{x}}=\frac{\mathrm{e}_{\mathrm{x}}}{2} \text { if } \mathrm{e} \in \varepsilon_{\mathrm{h}}^{\mathrm{i}}
$$

one gets

$$
\left(\mathrm{A}_{1}^{\mathrm{XY}}\right)_{\mathrm{ii}}=\sum_{\mathrm{e} \in \mathcal{E}_{\mathrm{h}}^{\mathrm{i}}} \frac{\mathrm{e}_{\mathrm{x}} \mathrm{e}_{\mathrm{y}}}{4}
$$

and

$$
\text { for } \mathrm{i} \neq \mathrm{j} \quad\left(\mathrm{A}_{1}^{\mathrm{XY}}\right)_{\mathrm{ij}}=\frac{\mathrm{e}_{\mathrm{X}} \mathrm{e}_{\mathrm{y}}}{4} \quad \text { if } \mathrm{e} \in \varepsilon_{\mathrm{h}}^{\mathrm{i}} \cap \varepsilon_{\mathrm{h}}^{\mathrm{j}}
$$

The entries of the other blocks of $\mathrm{A}_{1}$ can be easily deduced.

\section{Entries of the right hand side}

For each vertex $a_{i}$ of the mesh, let us introduce the associated set of edges: $\tilde{\varepsilon}_{\mathrm{h}}^{\mathrm{i}}=\left\{\mathrm{e} \in \varepsilon_{\mathrm{h}} /\right.$ edge

e and vertex $a_{i}$ belong to the same tetrahedron $\}$ and the set of elements: $\tau_{h}^{i}=$ support of $v_{i}$ $=\left\{\mathrm{T} \in \tau_{\mathrm{h}} / \mathrm{a}_{\mathrm{i}}\right.$ is a vertex of tetrahedron $\left.\mathrm{T}\right\}$ and for each edge $\mathrm{e} \in \varepsilon_{\mathrm{h}}, \tau_{\mathrm{h}}^{\mathrm{e}}=$ support of $\mathrm{W}_{\mathrm{e}}=\left\{\mathrm{T} \in \tau_{\mathrm{h}} / \mathrm{e}\right.$ is an edge of tetrahedron $\left.\mathrm{T}\right\}$. Then from (3),(9) and (15), it comes 


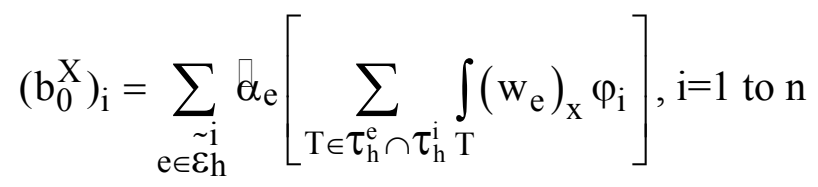

For $\mathrm{T} \in \tau_{\mathrm{h}}^{\mathrm{e}} \cap \tau_{\mathrm{h}}^{\mathrm{i}}$, the value of $\int_{\mathrm{T}}\left(\mathrm{w}_{\mathrm{e}}\right)_{\mathrm{x}} \varphi_{\mathrm{i}}$ can be computed as follows.

For $\mathrm{e}=\mathrm{ab}$ where $\mathrm{a}$ and $\mathrm{b}$ are vertices of tetrahedron $\mathrm{T}$ and denoting by $\lambda_{\mathrm{a}}, \lambda_{\mathrm{b}}$ the respective associated barycentric co-ordinates, one gets:

$$
\int_{\mathrm{T}}\left(\mathrm{w}_{\mathrm{e}}\right)_{\mathrm{x}} \varphi_{\mathrm{i}}=\frac{\operatorname{mes}(\mathrm{T})}{20} \eta \text { with } \eta=\left\{\begin{array}{l}
\left(\nabla \lambda_{\mathrm{b}}-2 \nabla \lambda_{\mathrm{a}}\right)_{\mathrm{x}} \text { if } \mathrm{e} \in \varepsilon_{\mathrm{h}}^{\mathrm{i}} \text { and } \mathrm{a}_{\mathrm{i}}=\mathrm{a} \\
\left(2 \nabla \lambda_{\mathrm{b}}-\nabla \lambda_{\mathrm{a}}\right)_{\mathrm{x}} \text { if } \mathrm{e} \in \varepsilon_{\mathrm{h}}^{\mathrm{i}} \text { and } \mathrm{a}_{\mathrm{i}}=\mathrm{b} \\
\left(\nabla \lambda_{\mathrm{b}}-\nabla \lambda_{\mathrm{a}}\right)_{\mathrm{x}} \text { if } \mathrm{e} \notin \varepsilon_{\mathrm{h}}^{\mathrm{i}}
\end{array}\right.
$$

Because of equality $\int_{e} E \cdot t=\alpha_{e}$, an explicit expression of the components of $b_{1}^{X}$ is more easily obtained from (9) and (16) as:

$$
\left(b_{1}^{\mathrm{X}}\right)_{\mathrm{i}}=\sum_{\mathrm{e} \in \varepsilon_{\mathrm{h}}^{\mathrm{i}}} \alpha_{\mathrm{e}} \int_{\mathrm{e}} \varphi_{\mathrm{i}} \cdot \mathrm{t}_{\mathrm{x}}=\sum_{\mathrm{e} \in \mathcal{\varepsilon}_{\mathrm{h}}^{\mathrm{i}}} \alpha_{\mathrm{e}} \frac{\mathrm{e}_{\mathrm{x}}}{2}
$$

\section{Limit $\operatorname{cases} \theta=0$ and $\theta=1$}

In the particular case where $\theta=0$, the linear system reduces to $\mathrm{A}_{0} \xi^{*}=\mathrm{b}_{0}$. As pointed out before, matrix $\mathrm{A}_{0}$ has a block diagonal structure with mass matrices on the diagonal. To save 
some computation time an explicit but approximate solution to the least squares problem can be obtained. Mass-lumping technique can be implemented to obtain an explicit solution of the least squares problem. The mass lumping allows to approximate the mass matrix by a diagonal matrix [Zienkiewicz, 1970]:

$$
\int_{\Omega} \mathrm{v}_{\mathrm{i}}^{2}=\frac{\beta}{4} \text { with } \beta=\sum_{\mathrm{T} \in \tau_{\mathrm{h}}^{\mathrm{i}}} \operatorname{mes}(\mathrm{T})
$$

The components of the approximate solution are given by:

$$
\left(\xi^{X}\right)_{i}=\frac{4}{\beta}\left(b_{0}^{X}\right)_{i} \quad \mathrm{i}=1 \text { to } n
$$

For $\theta=1$, the minimization problem defined by (2),(5) is a pure discrete least squares problem with one solution at least. The solutions satisfy the linear system $A_{1} \xi=S^{t} S \xi=b_{1}$ where $\mathrm{S}$ is the rectangular matrix defined by:

$$
\text { for } \mathrm{k}=1 \text { to cardinal }\left(\varepsilon_{\mathrm{h}}\right) \mathrm{S}_{\mathrm{ki}}=\int_{\mathrm{e}} \mathrm{v}_{\mathrm{i}} \cdot \mathrm{t} \mathrm{i}=1 \text { to } \mathrm{N}_{\mathrm{h}} \text { e edge of number } \mathrm{k}
$$

Uniqueness of the solution is ensured if there exists no $\xi \in \mathbb{R}^{N_{h}}-\{0\}$ such that $S \xi=0$. i.e. if the columns of $\mathrm{S}$ are linearly independent. It requires that the number of lines $\operatorname{card}\left(\varepsilon_{\mathrm{h}}\right)$ is larger than $\left(\mathrm{N}_{\mathrm{h}}\right)$ the number of columns.

From (27), the uniqueness condition of the solution can be also written as 
there exists no $E \in V_{h}-\{0\}$ such that $\int_{\mathrm{e}} \mathrm{E} . \mathrm{t}=0$ for any $\mathrm{e} \in \varepsilon_{\mathrm{h}}$

It can be translated into the following mesh characterization:

there is no vector field defined on the nodes such that $E(c) \neq 0$ for some node $c$ and $(E(a)+E(b)) \cdot e=0$ for any edge $e=a b$.

In the numerical experiments presented above such a property appears to be satisfied by the meshes. In particular, the necessary condition $\operatorname{card}\left(\varepsilon_{\mathrm{h}}\right) \geq \mathrm{N}_{\mathrm{h}}$ is always satisfied.

\section{Results and discussions}

In the following, method 1 (local averaging) is the reference method, method 2 (energy approximation) is obtained with $\theta=0$, method 3 (circulation approximation) corresponds to $\theta=1$, method 4 (combination of circulation approximation with energy approximation) is obtained for $\theta=1 / 2$, and method 5 corresponds to $\theta=0$ with the mass lumping approximation. For methods 2, 3, 4, a symmetric quasi-minimal residual method with SSOR preconditioning (PQMR) is used to solve the matrix system $\mathrm{A}_{\theta} \xi=\mathrm{b}_{\theta}$.

The accuracy and the time required for each post processing method are tested on 3 examples. In the first two examples, numerical results are compared with analytical solutions of scattering of a plane wave by a sphere. Analytical solutions can be found in [Harrington, 1968] and numerical formulations of scattering problems are given in [Yaobi, 1996]. A 50 mm radius - sphere is meshed with 100 nodes on its surface. The frequency of the incident plane wave is $1 \mathrm{GHz}$. Absorbing Boundary Condition (ABC) $\left(1^{\text {st }}\right.$ order Engquist Majda) is located at a half wavelength from the sphere. In order to estimate the influence of the Finite 
Element discretization, a relative error estimator on the sphere's surface (denoted by S) is computed as

$$
\text { Error }=\frac{\sum_{\mathrm{e} \in \mathrm{S}}\left|\alpha_{\mathrm{e}}^{\text {anal }}-\alpha_{\mathrm{e}}^{\text {num }}\right|}{\sum_{\mathrm{e} \in \mathrm{S}}\left|\alpha_{\mathrm{e}}^{\text {anal }}\right|}
$$

The edge values $\alpha_{\mathrm{e}}^{\text {num }}$ are those obtained from the FE-code. Denoting by $\mathrm{H}^{\text {anal }}$ the analytical solution to the magnetic formulation, the values $\alpha_{\mathrm{e}}^{\text {anal }}$ are approximations of $\int_{\mathrm{e}} \mathrm{H}^{\mathrm{anal}} . \mathrm{t}$ computed for an oriented edge $\mathrm{e}=\mathrm{ab}$ with nodes $\mathrm{a}$ and $\mathrm{b}$ as:

$$
\alpha_{\mathrm{e}}^{\mathrm{anal}}=\frac{\mathrm{H}^{\mathrm{anal}}(\mathrm{a})+\mathrm{H}^{\mathrm{anal}}(\mathrm{b})}{2} \cdot \frac{\mathrm{e}}{|\mathrm{e}|}
$$

The efficiency of the different post processing strategies is also evaluated on the surface of the sphere by means of relative error estimators. For each of the five methods, 3 relative errors concerning the nodal vector field, its normal component and its tangential component are computed as

$$
\text { Error }=\frac{\sum_{\mathrm{a} \in \mathrm{S}}\left|\mathrm{H}^{\mathrm{anal}}(\mathrm{a})-\mathrm{H}^{\text {num }}(\mathrm{a})\right|}{\sum_{\mathrm{a} \in \mathrm{S}}\left|\mathrm{H}^{\mathrm{anal}}(\mathrm{a})\right|}
$$


Where $\mathrm{H}$ indicates either the vector (3 components), or its normal component or its tangential component. Nodal values $\mathrm{H}^{\text {num }}$ (a) are given by the post processing methods from the F.E. edge values $\alpha_{\mathrm{e}}^{\text {num }}$.

\section{Scattering by a perfect electric conductor sphere:}

In the first example (figure 1), the sphere is modeled as a perfect electrical conductor (PEC). The problem is meshed with 4481 nodes and 22108 tetrahedral elements leading to 28161 edges (294 edges on the sphere's surface). The edge values error, computed with (28), is about 0.067 . The total solving time (assembling and solving of the matrix system) with the FE code is about 300 s on a HP J5000. Note that the same solver as in the post processing is used (217 iterations of PQMR).

Figure 1

Various relative errors are given in Table I which also contains the post processing costs.

Table I: relative errors (30), number of iterations of the PQMR and CPU time for the post processing for the PEC sphere.

\begin{tabular}{|c|c|c|c|c|c|}
\hline Method & Relative error on 3 & Relative error on & Relative error & Iterations & Time \\
components & tangential & on normal & & \\
\hline 1 & 0.238 & 0.120 & 0.200 & $/$ & 2 \\
\hline 2 & 0.190 & 0.080 & 0.173 & 7 & 11 \\
\hline 3 & 0.130 & 0.072 & 0.100 & 22 & 21 \\
\hline 4 & 0.133 & 0.069 & 0.113 & 19 & 20 \\
\hline
\end{tabular}




\begin{tabular}{|l|l|l|l|l|l|}
\hline 5 & 0.255 & 0.161 & 0.200 & $/$ & 1 \\
\hline
\end{tabular}

On this first example, the methods with circulation approximation (mixed with energy approximation in method 4, pure in 3) give close results and appear to be the most accurate. From the reference method (method 1) the increase in precision on the nodal vector field is about 0.1 . The increase in time (about $21 \mathrm{~s}$ ) is reasonable as compared with the time required to solve the FE problem (436 s). With method 2 (pure energy approximation), the accuracy gain is roughly divided by $2(0.048)$ and disappears when the mass lumping is introduced (method 5). The costs behave in similar ways.

\section{Scattering by a magnetic sphere:}

In the second example, the sphere is magnetic with $\mu_{\mathrm{r}}=3$. A discontinuity of the magnetic field at the surface of the sphere is therefore introduced and the interior (region 1) has to be meshed. The same mesh as for the PEC sphere (figure 1) is used outside of the sphere (region 2). For the whole mesh, one gets 4547 nodes and 22783 tetrahedral elements leading to 28805 edges (294 edges on the sphere's surface). The total time for FE solving is about $436 \mathrm{~s}$ (537 iterations of the PQMR). The edge values error, computed with (28), is about 0.149 . The post processing is made in the only region 2 (air) according to the analytical solution (Harrington, 1968).

Similarly as in Table I, Table II gives the results we obtain for each five methods. Compared to the reference method, the least squares methods behave as in the PEC case.

Table II: relative errors (30), number of iterations of the PQMR and CPU time for the post processing for the magnetic sphere. 


\begin{tabular}{|c|c|c|c|c|c|}
\hline Method & $\begin{array}{c}\text { Relative error on 3 } \\
\text { components }\end{array}$ & $\begin{array}{c}\text { Relative error on } \\
\text { tangential } \\
\text { component }\end{array}$ & $\begin{array}{c}\text { Relative error } \\
\text { on normal } \\
\text { component }\end{array}$ & Iterations & Time \\
\hline 1 & 0.265 & 0.133 & 0.230 & $/$ & 2 \\
\hline 2 & 0.188 & 0.098 & 0.163 & 7 & 9 \\
\hline 3 & 0.127 & 0.092 & 0.087 & 20 & 19 \\
\hline 4 & 0.129 & 0.089 & 0.090 & 18 & 19 \\
\hline 5 & 0.289 & 0.129 & 0.260 & $/$ & 1 \\
\hline
\end{tabular}

\section{Academic example:}

In order to eliminate the error due to FE discretization, the performances of the post processing methods are finally evaluated on an academic example on the same spherical geometry. The same geometry and the same mesh as for the problem of scattering by the dielectric sphere (example 2) are used. The vector field to post process is no longer the solution to some scattering problem but a radial field analytically defined as (figure 2):

$$
20 \mathrm{r} \text { in region } 1 ; 2000 \mathrm{r} \text { in region } 2
$$

with $\mathrm{r}$ the radial component in spherical coordinates $(\mathrm{r}, \theta, \varphi)$.

Figure 2

Approximate edge values are then evaluated as in (29). From these data, nodal values are computed with the different post processing methods independently in both regions (table III). 
Table III: nodal values, number of iterations of the PQMR of the post processing for the academic problem.

\begin{tabular}{|c|c|c|c|c|c|}
\hline Method & Normal & Normal & Tangential & Tangential & Iterations \\
component & component & component & component & \\
\hline 1 & 1.2 & 71 & 0.019 & 0.081 & \\
\hline 2 & 1.1 & 87 & 0.002 & 0.177 & 8 \\
\hline 3 & 1.0 & 100 & $3.10^{-14}$ & $8.10^{-12}$ & 23 \\
\hline 4 & 1.0 & 98 & $1.10^{-5}$ & 0.004 & 20 \\
\hline 5 & 1.2 & 70 & 0.003 & 0.108 & \\
\hline
\end{tabular}

Taking into account the starting field (31), the awaited results on the sphere surface should be :

region 1: tangential component $=0$; normal component $=1$

region 2 : tangential component $=0 ;$ normal component $=100$

The results confirm an important increase of accuracy obtained with pure or mixed circulation approximation methods. In particular exact results are given by method 3 .

\section{Conclusion}

As shown from tables I, II and III, the most accurate vector fields are obtained with the methods where some circulation approximation is satisfied. Theses methods are the most CPU consuming. However, the time required for the post processing remains reasonable compared 
to the total FE solving time. A significant improvement of the accuracy is so obtained with a small additional computation time.

\section{References}

Dibben, C. D., Metaxas, R., (1997), "A Comparison of Errors Obtained with Withney and Linear Edge Elements," IEEE Transaction on Magnetics, vol. 33, No. 2, pp. 1524-1527.

Harrington, R. F., (1968), Field Computation by Moment Methods, MacMillan, New-York.

Sekkak, A., Pichon, L., Razek, A. (1994), "3-D FEM Magneto-Thermal Analysis in Microwave Ovens," IEEE Transaction on Magnetics, vol. 30, No 5, pp. 3347-3350.

Volakis, L. J., Davdson, D. B., (2000) "Implementation Issues for Three Dimensional Vector FEM Programs", IEEE Antennas and Propagation Magazine, vol. 42, No. 6, pp. 100-107.

Webb, J. P. (1993), "Edge element and what they can do for you," IEEE Transaction on Magnetics, vol. 29, No. 2, pp. 1460-1465.

Yao Bi, J. L., Nicolas, L., Nicolas, A (1996), "Vector absorbing boundary conditions for nodal or mixed finite elements," IEEE Transaction on Magnetics, vol. 32, No 3, pp.848-853.

Zhao, H., Turner, I. W. (2000), "The Use of Coupled Computation Model for Studying the Microwave Heating of Wood," Applied Mathematical Modelling, No 24, pp. 183-197.

Zienkiewicz, (1970), O. C., The Finite Element Method, Mc Graw-Hill Book Company.

\section{Figures}




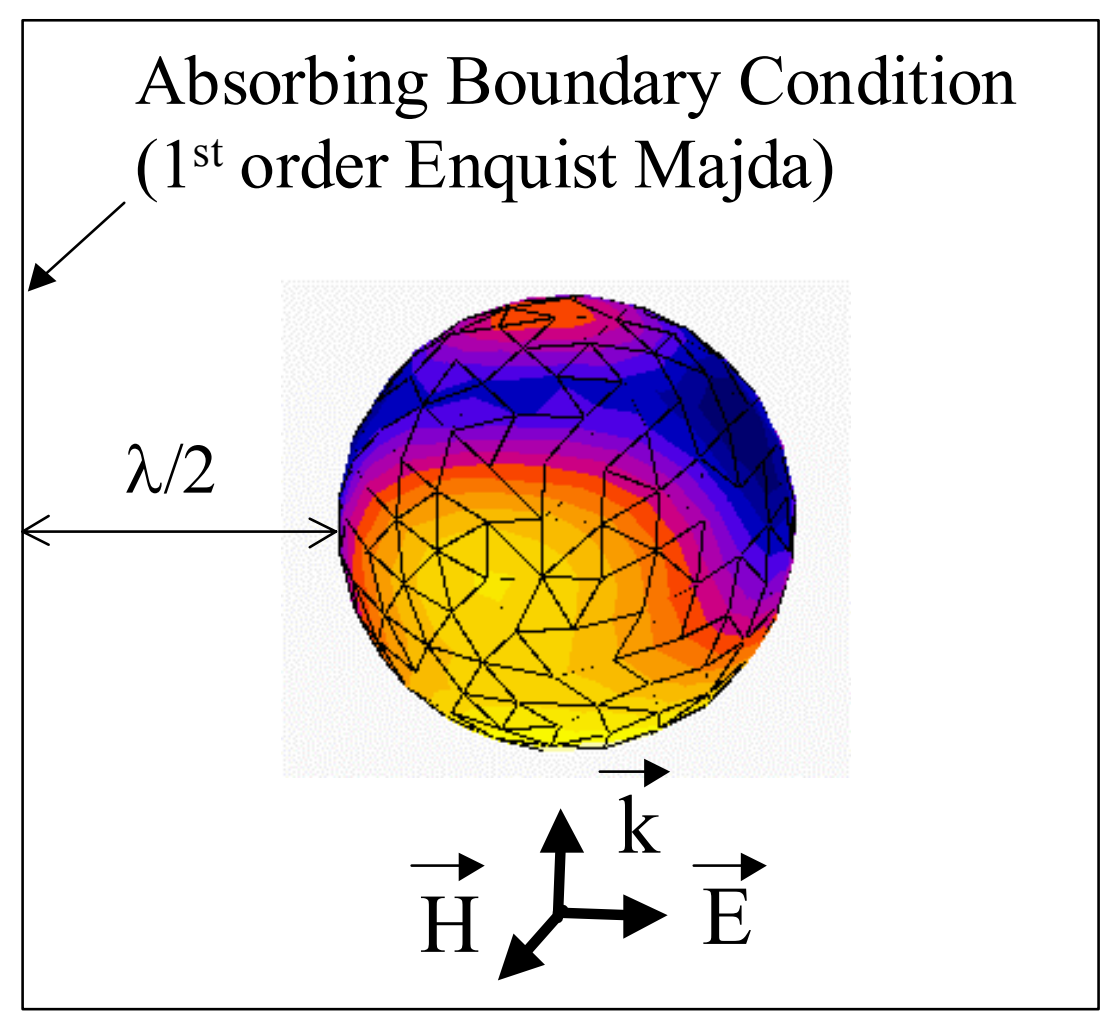

Figure $1: \mathrm{H}$ field modulus on a PEC sphere

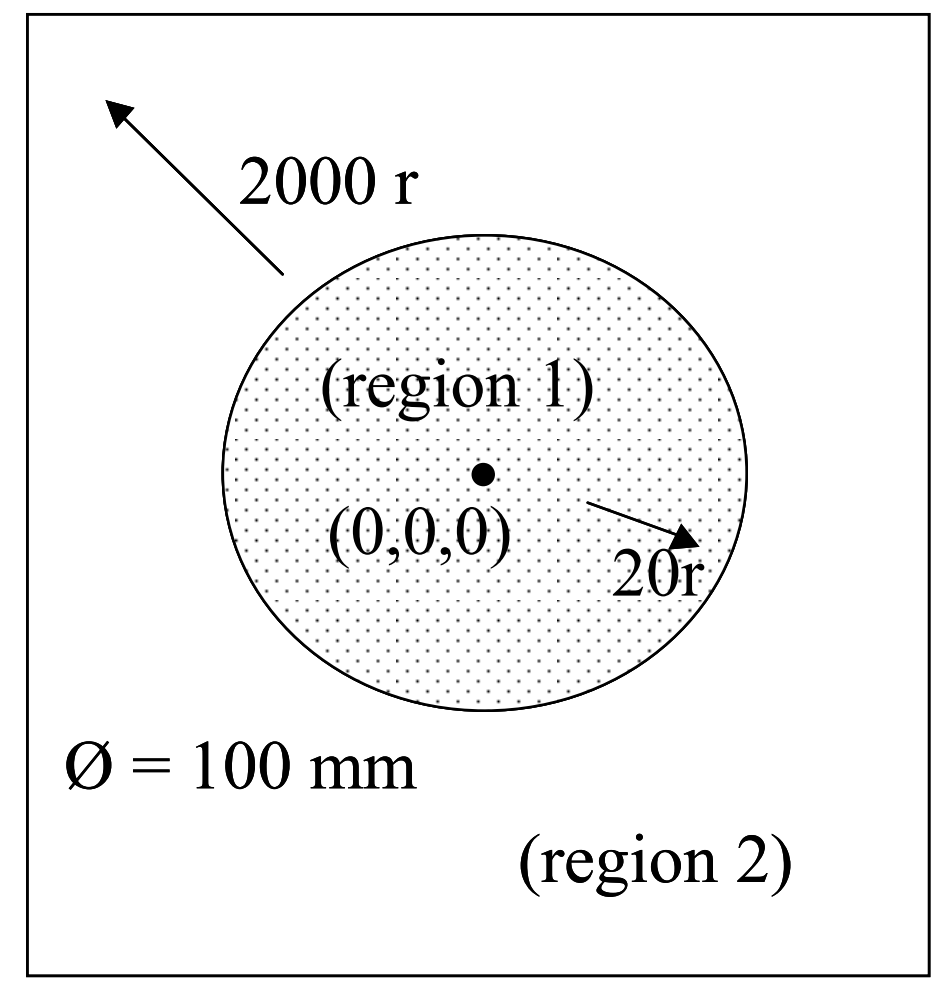

Figure 2 : radial field of magnitude 20r in region 1 and 2000r in region 2 